\title{
Utilization Strategy Research Of Goafs Based On Correspondence Analysis
}

\author{
Shiyao Xiang ${ }^{1, a}$, Mingdong Zhao ${ }^{1, b}$, Dongjing $\mathrm{Xu}^{2, \mathrm{c}}$ and Yijin Chen ${ }^{1, \mathrm{~d}}$ \\ ${ }^{1}$ College of Geoscience and Surveying Engineering, China University of Mining and Technology \\ (Beijing), Beijing 100083, China \\ ${ }^{2}$ State Key Laboratory of Coal Resources and Safe Mining, China University of Mining and \\ Technology (Beijing), Beijing 100083, China

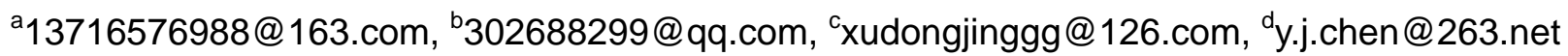

Keywords: Goafs utilization; Coal mine; Collapse rules; Correspondence analysis

Abstract. According to the energy development policy and energy structure adjustment in China, this paper puts forward the utilization strategy of goafs based on correspondence analysis. Firstly, the correspondence analysis was used to discriminate the goafs with hidden collapse risk and research the collapse rules in coal mine. Using this method, 7 variables which affected the collapse of coal mine goaf and 24 samples were analyzed by R factor analysis and Q factor analysis to complete the distribution picture of variables and samples. The variables included degree of complex geological structure, goaf volume rate, vertical depth away from the surface, dip angle of coal seam, number of spatial superimposed layers, overburden thickness and overburden type. The analyses were conducted by the distribution picture to research the collapse rule of goafs. The corresponding measures could be taken to treat the goafs according to collapse rule. Secondly, the projects of establishing underground water storage system, underground oil and gas storage system and underground waste disposal system were proposed to realize the goafs utilization.

\section{Introduction}

Coal is main energy in China. With the energy structure adjustment, maladjustment of coal supply and demand has appeared in recent years. The coal supply is far more than the demand of market. Coal enterprises get into trouble because of unmarketable coal. National Energy Administration of China says that the government should strictly limit new capacity of coal. In principle, the examination and approval of new coal mine project should be suspended within 3 years from 2016 . The backward production facilities in the coal industry should be closed down and the number of the closed backward coal mines of which the total capacity is 60 million tons should be more than 1000 in 2016. From the beginning of reform and opening-up to 2008, Shanxi Province has produced nearly 10 billion tons raw coal. At the same time, 6.3 billion cubic meters of goafs have generated and the area of the goafs is about 5115 square kilometers. Problems such as ground collapse, ground fissure and landslide have appeared. A large number of cultivated land and forest land have been destroyed. The energy development and utilization within the environmental capacity is really reasonable, clean and scientific. Coordinated development between coal exploitation and environmental protection is the urgent request of new energy situation. In the past decades, the goafs produced by coal mining have been a great safe hidden trouble. The disasters caused by goafs can be mainly divided into 4 types: water inrush, fire, toxic and harmful gas emission and geological hazards [1]. According to problems mentioned above, this paper proposes the utilization strategy of goafs. The correspondence analysis has been used to discriminate the goafs with hidden collapse risk and research the collapse rules. Then, this paper introduces how to make value by utilizing goafs.

\section{Evaluation Methods of Goafs Collapse Rules}

Goaf collapse which seriously threats the life and property safety of the residents in mining areas belongs to the geological hazards caused by goafs. Determining the goafs with hidden collapse risk and figuring out the collapse rules have great significance for goafs management. 
Detecting goafs and finding out the goafs with hidden collapse risk by geophysical prospecting methods [2] is one of the common methods. Using existing data to discriminate the goafs with hidden collapse risk and research the collapse rules by statistical analysis methods have been generated, as the accumulation of geological data. For example, distance discriminant forecast method [3], catastrophe progression method [4] and FLAC ${ }^{3 \mathrm{D}}$ numerical analysis method [5] have been used. In order to discriminate the goafs with hidden collapse risk and research the collapse rules, this paper puts forward the correspondence analysis method based on statistical analysis to study the characteristics of goaf collapse.

Algorithm Principle. The correspondence analysis method is a statistical method on the basis of factor analysis. It connects $\mathrm{R}$ factor analysis (variable) with $\mathrm{Q}$ factor analysis (sample) through transition matrix $\mathbf{Z}$. By the results of $\mathrm{R}$ factor analysis, the results of $\mathrm{Q}$ factor analysis can be obtained to solve the computational difficulties of large numbers of samples. In the end, the variables and samples are reflected on the same factor plane to reveal the relevance of each variable and each sample as well as that between variables and samples.

Assume the raw data has $n$ samples and $p$ variables, and organize the data as Eq. 1:

$$
\boldsymbol{X}=\left[x_{i j}\right]=\left[\begin{array}{cccc}
x_{11} & x_{12} & \cdots & x_{1 p} \\
x_{21} & x_{22} & \cdots & x_{2 p} \\
\vdots & \vdots & & \vdots \\
x_{n 1} & x_{n 2} & \cdots & x_{n p}
\end{array}\right](i=1,2, \cdots, n ; j=1,2, \cdots, p)
$$

Step 1: Calculate the sum of each row, each column and all elements of matrix $\boldsymbol{X}$.

$x_{i \cdot}=\sum_{j=1}^{p} x_{i j}, \quad x_{\cdot j}=\sum_{i=1}^{n} x_{i j}, \quad T=\sum_{i=1}^{n} \sum_{j=1}^{p} x_{i j}(i=1,2, \cdots, n ; j=1,2, \cdots, p)$

Step 2: Calculate the transition matrix $\mathbf{Z}=\left[z_{i j}\right]_{n \times p}$. And $z_{i j}$ can be written as Eq. 3:

$$
z_{i j}=\frac{x_{i j}-x_{i \cdot} \cdot x_{\cdot j} / T}{\sqrt{x_{i} \cdot x_{\cdot j}}}(i=1,2, \cdots, n ; j=1,2, \cdots, p)
$$

Step 3: Do R factor analysis.

Calculate the characteristic roots which include $\lambda_{1}, \lambda_{2} \cdots \lambda_{p}$ by covariance matrix $\boldsymbol{A}=\boldsymbol{Z}^{T} \boldsymbol{Z}$, and order them in decreasing order, such as $\lambda_{1} \geq \lambda_{2} \geq \cdots \lambda_{p}$. Select the first $m$ characteristic roots whose cumulative contribution rate $\left(\sum_{k=1}^{m} \lambda_{k} / \sum_{i=1}^{p} \lambda_{i}\right) \geq 85 \%$ and calculate the corresponding unit characteristic vectors which include $\boldsymbol{u}_{1}, \boldsymbol{u}_{2} \cdots \boldsymbol{u}_{m}$. The $\mathrm{R}$ factor loading matrix can be calculated as Eq. 4:

$$
\boldsymbol{F}=\left[\begin{array}{cccc}
u_{11} \sqrt{\lambda_{1}} & u_{12} \sqrt{\lambda_{2}} & \cdots & u_{1 m} \sqrt{\lambda_{m}} \\
u_{21} \sqrt{\lambda_{1}} & u_{22} \sqrt{\lambda_{2}} & \cdots & u_{2 m} \sqrt{\lambda_{m}} \\
\vdots & \vdots & & \vdots \\
u_{p 1} \sqrt{\lambda_{1}} & u_{p 2} \sqrt{\lambda_{2}} & \cdots & u_{p m} \sqrt{\lambda_{m}}
\end{array}\right]
$$

Step 4: Do Q factor analysis.

Since matrix $\boldsymbol{B}=\mathbf{Z Z} \mathbf{Z}^{T}$ and matrix $\boldsymbol{A}=\boldsymbol{Z}^{T} \boldsymbol{Z}$ have the same characteristic roots, the unit characteristic vectors which include $\boldsymbol{v}_{1}=\mathbf{Z} \boldsymbol{u}_{1} / \sqrt{\lambda_{1}}, \boldsymbol{v}_{2}=\mathbf{Z} \boldsymbol{u}_{2} / \sqrt{\lambda_{2}}, \cdots, \boldsymbol{v}_{m}=\mathbf{Z} \boldsymbol{u}_{m} / \sqrt{\lambda_{m}}$ of matrix $\boldsymbol{B}$ can be obtained by the calculated characteristic roots and unit characteristic vectors of matrix $\boldsymbol{A}$. The $\mathrm{Q}$ factor loading matrix can be calculated as Eq. 5: 


$$
\boldsymbol{G}=\left[\begin{array}{cccc}
v_{11} \sqrt{\lambda_{1}} & v_{12} \sqrt{\lambda_{2}} & \cdots & v_{1 m} \sqrt{\lambda_{m}} \\
v_{21} \sqrt{\lambda_{1}} & v_{22} \sqrt{\lambda_{2}} & \cdots & v_{2 m} \sqrt{\lambda_{m}} \\
\vdots & \vdots & & \vdots \\
v_{n 1} \sqrt{\lambda_{1}} & v_{n 2} \sqrt{\lambda_{2}} & \cdots & v_{n m} \sqrt{\lambda_{m}}
\end{array}\right]
$$

Step 5: Complete the distribution picture of variables and samples on the same factor plane by $\mathrm{R}$ factor loading matrix and Q factor loading matrix.

Step 6: Analyze the relevance of each variable and each sample as well as that between variables and samples by the distribution picture.

Application Example and Analysis. This paper uses the goaf collapse data of Beijing Western Hills as research data from reference [6]. The coal mining of this region has been done for a long time and the surface and shallow underground coal has been exhausted. Large and multi-levels goafs have been generated in deep underground. Through field investigation, the results show that the influence factors of causing goaf collapse are as follows: degree of complex geological structure, goaf volume rate, vertical depth away from the surface, dip angle of coal seam, number of spatial superimposed layers, overburden thickness and overburden type. We number them in sequence as $X_{1}-X_{7}$ and use them as the variables for correspondence analysis. 24 samples with clear statuses which can be divided into collapsed type and stable type in this region have been selected. The 1-17 samples are the samples that have collapsed or are in stable status and the 18-24 samples are the samples whose statuses should be discriminated.

By arranging and calculating the data of reference [6] and doing $\mathrm{R}$ factor analysis, the characteristic roots of matrix $\boldsymbol{A}$ can be obtained and the contribution rates and cumulative contribution rates of each characteristic root can also be calculated. The first two characteristic roots are shown in Table 1.

Table 1. Characteristic roots, contribution rates and cumulative contribution rates

\begin{tabular}{ccc}
\hline Characteristic $\operatorname{Root}\left[\lambda_{i}\right]$ & Contribution Rate[\%] & Cumulative Contribution Rate[\%] \\
\hline 0.0550 & 63.73 & 63.73 \\
0.0223 & 25.84 & 89.57 \\
\hline
\end{tabular}

The cumulative contribution rate of $\lambda_{1}$ and $\lambda_{2}$ has reached $89.57 \%$, so the two factors are able to reflect the basic information of each variable well. The $\mathrm{R}$ factor loading matrix can be obtained by the characteristic roots of matrix $\boldsymbol{A}$. According to the relevance between $\mathrm{Q}$ factor analysis and $\mathrm{R}$ factor analysis, the $\mathrm{Q}$ factor loading matrix can be further obtained. The distribution picture of variables and samples can be completed by $\mathrm{R}$ factor loading matrix and $\mathrm{Q}$ factor loading matrix.
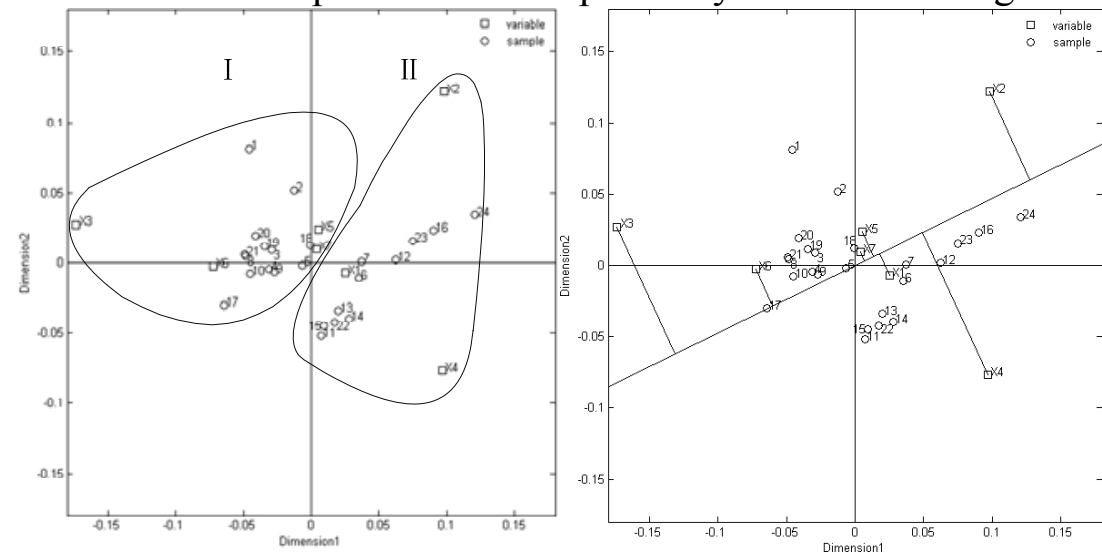

Fig. 1 Distribution picture of variables and samples Fig. 2 Influence degree analysis

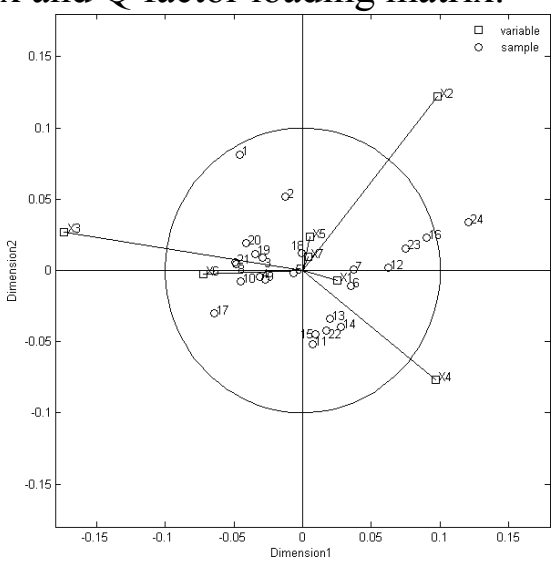

Fig. 3 Relevance analysis

a) The classification analysis

In Fig. 1, squares represent the variables and circles represent the samples. According to the graphic density, they can be divided into two categories. The numbers of samples belonging to the first category are $1,2,3,4,5,8,9,10,17,18,19,20$ and 21 . The numbers of variables belonging to the first category are $X_{3}, X_{5}, X_{6}$ and $X_{7}$. The numbers of samples belonging to the second category are $6,7,11,12,13,14,15,16,22,23$ and 24 . The numbers of variables belonging to the second 
category are $X_{1}, X_{2}$ and $X_{4}$. The samples of the first category all belong to the collapsed type and the main influence factors are the vertical depth away from the surface, number of spatial superimposed layers, overburden thickness and overburden type. The samples of the second category all belong to the stable type and the main influence factors are the degree of complex geological structure, goaf volume rate and dip angle of coal seam. The classification results are in accordance with the actual situation. The purpose of discriminating the type of unknown samples is realized through the known samples that belong to the same category. Compared with actual situation, the correct rate of overall classification is very high, and the goafs with hidden collapse risk have been discriminated accurately, and the main influence factors of each type have been clarified.

b) The influence degree analysis

Connect the origin with NO.17 sample and extend both ends of the line. Paint vertical lines from variables to the line and record the locations of pedals. As shown in Fig. 2. The degrees of each variable affecting NO.17 sample can be obtained by comparing the distances between each pedal and NO.17 sample. The distance is shorter, the relevance is closer. In this way, the influence degree is greater. The variables can be ranked in decreasing order by the influence degree as the overburden thickness, overburden type, vertical depth away from the surface, number of spatial superimposed layers, degree of complex geological structure, dip angle of coal seam and goaf volume rate. The corresponding results can be got by connecting the origin with other sample. Similarly, the influence degrees between the variable and each sample can be obtained by connecting the origin with variables.

c) The relevance analysis

Connect the origin with each variable and paint a circle with the origin as center and a certain length as radius. As shown in Fig. 3. The relevance between each variable can be obtained by comparing the angles between each other and calculating by cosine law. The angle is smaller, the relevance is closer. In this way, the variables play a similar role. The relevance between number of spatial superimposed layers and overburden type are the most closely and they play a similar role in affecting goaf collapse. The relevance between vertical depth away from the surface and dip angle of coal seam are the remotest and they play different roles in affecting goaf collapse. Similarly, the relevance between each sample can be obtained by connecting the origin with each sample. So the similarity and dissimilarity of samples can also be got. The circle centering by the origin shows that goaf volume rate, vertical depth away from the surface and dip angle of coal seam are far away from the center of circle. So, we can get that the three variables have big differences with the others.

\section{Goafs Utilization}

Huge underground space has been generated by goafs. Targeted treatment by researching the collapse rules and analyzing the main influence factors can realize goafs utilization to make value. In this paper, the following aspects have introduced the goafs utilization.

a) Underground water storage system

Coal mining will destroy the groundwater system, and thus a large amount of mine water has been produced. At the same time, the water resource in main coal producing areas is mostly scarce. The utilization of mine water to solve water consumption of mine area has become an important research subject. If a large number of mine water was stored on the surface, on the one hand a lot of ground space would be taken up, on the other hand a large amount of water would be consumed due to evaporation. Using the underground space of goafs and reinforcing the security coal pillar can build reservoir through connecting to artificial dam. Using the purification effect of rock mass and installing water purifiers can realize the storage and purification of mine water. In this way, the needs of water in mining area could be satisfied [7]. Underground water storage system can make full use of existing underground space and occupy little land resources; avoid flooding, dam break, siltation and other issues; reduce the evaporation loss and protect water resources effectively, etc.

b) Underground oil and gas storage system

Through the special seepage prevention of underground goafs, oil and natural gas can be stored in the goafs to make full use of underground space, on the premise of avoiding oil and natural gas 
leak. Finland and America have established underground fuel depots to store oil and fuel [8]. Storing oil and gas in goafs can avoid excavating special underground storage space and make efficient use of existing underground resources.

c) Underground waste disposal system

Every year China will generate a large number of industrial and living garbage. The garbage should be dealt with to reduce the harm to environment. The garbage storage and processing are usually carried out on the surface, which will occupy a lot of valuable land resources and cause air pollution at the same time. It will seriously affect the normal life of surrounding residents. Underground waste disposal system can effectively solve the problem of land use and environmental pollution by reusing the abandoned goafs. The garbage which must be dealt with by burying can be buried in the goafs through professional processing.

\section{Conclusions}

(1)The discrimination of the goafs with hidden collapse risk has been realized by correspondence analysis through the distribution picture of variables and samples and the correct rate is very high. The relevance of each variable and each sample as well as that between variables and samples can be made clear by the distribution picture of variables and samples. So the collapse rules of goafs can be obtained.

(2)According to the collapse rules of goafs obtained by correspondence analysis, the corresponding measures can be taken to govern goafs to achieve further utilization of goafs. The goafs utilization can reduce damage to the environment of coal mining and make use of the underground space effectively to suit the current coal and energy situation.

\section{Acknowledgements}

The authors gratefully acknowledge the financial support of the Fundamental Research Funds for the Central Universities (No. 2010YD06).

\section{References}

[1] W. Li, and J. Li: Goaf Disaster Characteristics of Resources Integration Coal Mines and Their Control Countermeasures, Saf. Coal Mine., Vol. 46 (2015), p. 179

[2] S.B. Li, Z.J. Li, Y. Zhang, and H.J. Lu: Application of Acoustic Emission Monitoring Techniques in Geostress Hazard Prediction of Mined-out Areas, Met. Mine, No. 3 (2014), p. 152

[3] G.J. Peng, Y.H. Fu, and L.J. Dong: A Distance Discriminant Analysis Method for The Prediction of Goaf Collapse, Nonf. Met. (Min. Sect.), Vol. 61 (2009), p. 50

[4] H.J. Chen, X.B. Li, K. Gao: On The Application of Catastrophe Progression Method to Predicting The Likely Mining Collapse Accidents, J. Saf. Env., Vol. 8 (2008), p. 108

[5] H.P. Yuan, J.L. Wang, K. Zhao, K. Zhao, and Y.J. Zhai: Study on The Goaf Form Influence on Distribution of Surface Subsidence, Met. Mine, No. 11 (2011), p. 25

[6] N.Q. Shen, J.W. Yang, and X.P. Zheng: Prediction of Mining Collapse Based on Neural Network, Coal Geol. Explor., Vol. 29 (2001), p. 42

[7] D.Z. Gu: Theory Framework and Technological System of Coal Mine Underground Reservoir, J. China Coal Soc., Vol. 40 (2015), p. 239

[8] J.P. Li, C.G. Feng, and Q.X. Zeng: A Review of The Utilization of Mined Area, Met. Mine, No. 10 (2002), p. 4 